\title{
Acromioclavicular Joint Injuries: Effective Rehabilitation
}

\author{
Matthew R LeVasseur (D) \\ Michael R Mancini (ID) \\ Daniel P Berthold ${ }^{1,2}$ \\ Antonio Cusano' \\ Grace P McCann' \\ Mark P Cote' \\ Gregg Gomlinski \\ Augustus D Mazzocca' \\ 'Department of Orthopaedic Surgery, \\ University of Connecticut, Farmington, \\ CT, USA; ${ }^{2}$ Department of Orthopaedic \\ Sports Medicine, Technical University of \\ Munich, Munich, Germany
}

Purpose: To perform a review of the literature focusing on rehabilitation protocols in patients with acromioclavicular (AC) joint injuries treated operatively and nonoperatively and to provide an updated rehabilitation treatment algorithm.

Methods: Studies were identified by searching the MEDLINE database from 01/1995 to 09/ 2020. Included studies contained detailed rehabilitation protocols with physiologic rationale for AC joint injuries. Biomechanical studies, technique articles, radiographic studies, systematic reviews, case studies, editorials, and studies that compared nonoperative versus operative treatment without focus on rehabilitation were excluded. Following identification of the literature, an updated treatment algorithm was created.

Results: The search strategy yielded 1742 studies, of which 1654 studies were excluded based on title, 60 on the abstract, and 25 on the full manuscript. One study was manually identified using article reference lists, yielding four publications presenting detailed rehabilitation protocols based on physiologic rationale. No randomized controlled trials or comparative studies were identified or cited as a basis for these rehabilitation protocols.

Conclusion: Few detailed rehabilitation protocols in patients with AC joint injuries have been published. These protocols are limited by their standardization, arbitrary timelines, and provide minimal assessment of individual patient characteristics. The quality of patient care can be improved with more practical guidelines that are goal-oriented and allow for critical thinking among clinicians to address individual patient needs. Three common barriers preventing successful rehabilitation were identified and addressed: Pain, Apprehension, and (anterior chest wall) Stiffness to regain Scapular control, effectively termed "PASS" for AC joint rehabilitation.

Clinical Relevance: Rehabilitation protocols for AC joint injuries should be less formulaic and instead allow for critical thinking and effective communication among clinicians and therapists to address individual patient needs.

Keywords: acromioclavicular joint, AC joint, rehabilitation, physical therapy

\section{Introduction}

Acromioclavicular (AC) joint injuries are common in young athletes and are one of the most prevalent shoulder injuries in contact sports. ${ }^{1}$ Reports have shown that $41 \%$ of collegiate football players and $40 \%$ of National Football League quarterbacks experience AC joint dislocations during their careers. ${ }^{2,3}$ Additionally, AC joint injuries have also been observed in high numbers during noncontact sports such as cycling or triathlon. ${ }^{4}$ The most common mechanism of injury is a direct force to the shoulder during a fall with the arm in an adducted position. ${ }^{5-7}$ Historically, these injuries have been classified using the Rockwood classification, in which Types I and II are treated nonoperatively and Types IV-VI are generally
Correspondence: Matthew R LeVasseur Email mlevasseur@uchc.edu 
treated operatively. ${ }^{5}$ However, there is significant controversy regarding Type III injuries, with many practitioners opting for a trial of nonoperative therapy and reserving surgical intervention for those who remain symptomatic. Furthermore, there have been many recent investigations and comprehensive reviews comparing operative and nonoperative management for patients with Type III injuries. ${ }^{8-10}$ However, the role of physical therapy and effective rehabilitation during definitive nonoperative care or following surgical intervention has yet to be substantiated.

Nonoperative modalities of care following AC joint injuries include rest, non-steroidal anti-inflammatory medications, local anesthetics, kinesiology tape, and various forms of rehabilitation. Some authors have demonstrated satisfactory outcomes of lower grade injuries by allowing patients to increase activities as tolerated, without strict rehabilitation. ${ }^{11,12}$ Contrarily, the prevalence of scapular dyskinesis following AC joint injury suggests that physical therapy and rehabilitation have a significant role to address the ensuing muscular imbalance. ${ }^{13-15}$ However, best practice guidelines considering rehabilitation protocols for $\mathrm{AC}$ joint injuries, are yet to be clinically validated. ${ }^{16}$ The role of rehabilitation is further blurred by an abundance of surgical techniques and differing rehabilitation protocols amongst surgeons. Moreover, there are no randomized controlled trials comparing different rehabilitation protocols, for both operative and nonoperative care. Additionally, there are limited studies attempting to evaluate the efficacy of a robust rehabilitation protocol.

The purpose of this study is to perform a systematic review of the literature in regards to rehabilitation protocols for operative and nonoperative management of acromioclavicular joint injuries that are based on physiologic rationale. The presented data will be used to portray current deficiencies in rehabilitation protocols. The authors will present their updated rehabilitation algorithm to highlight patient-specific obstacles.

\section{Methods}

\section{Identification of Studies}

Two investigators (MRL, MRM) conducted a search of the MEDLINE database in October 2020 using the combination of search terms: (acromioclavicular OR "AC joint" OR ACJ) AND (rehabilitation OR nonoperative OR conservative OR surgical OR operative). Broad search terms were utilized to identify all applicable studies. Search limits were English language and studies from January 1, 1995 to September 30, 2020. Dates were chosen to allow inclusion of the earliest study known to the authors. ${ }^{17}$ Studies were included if they presented detailed rehabilitation protocols based on physiologic rationale for operative or nonoperative care. Studies were excluded if they met the following criteria: compared nonoperative versus operative treatment without a focus on rehabilitation, biomechanical studies, technique articles, radiographic studies, systematic reviews, case studies, or editorials.

Studies were uploaded into a literature review program and screened in the following order by two investigators (MRL, MRM): title, abstract, and then full manuscript. Disagreements regarding inclusion were resolved with consensus. For each included study, the following information was collected: indication, physiologic rationale or evidence, and protocol.

During the screening of eligible studies, no randomized controlled trials were identified. As such, the Cochrane Risk of Bias tool was not utilized. Furthermore, a metaanalysis was unable to be performed. To ensure all studies of interest were appropriately compiled, the "related articles" feature in MEDLINE and the reference lists of included studies were utilized to identify any additional studies meeting the inclusion criteria.

\section{Results}

Using the search term criteria, 1742 studies were identified on the MEDLINE database. Of these, 1654 studies were excluded based on the title, 60 studies were excluded based on the abstract, and 25 studies were excluded based on the full manuscript. Using the "related articles" feature and reference lists of included studies, one additional article was identified that met all criteria for inclusion. This resulted in a final group of 4 studies. No randomized controlled trials were identified investigating various forms of rehabilitation. The flowchart is shown in Figure 1.

\section{Rehabilitation Protocols}

The literature review identified 4 studies with extensive rehabilitation protocols. Two studies addressed rehabilitation of AC joint injury (distinguished by injury type) and two studies addressed rehabilitation for scapular dyskinesis. These studies are shown in Tables 1 and 2. None of these studies had clinical validation at the time of publication. Importantly, these protocols are rigid and exhaustive with arbitrary timelines, which limit their clinical utility. 


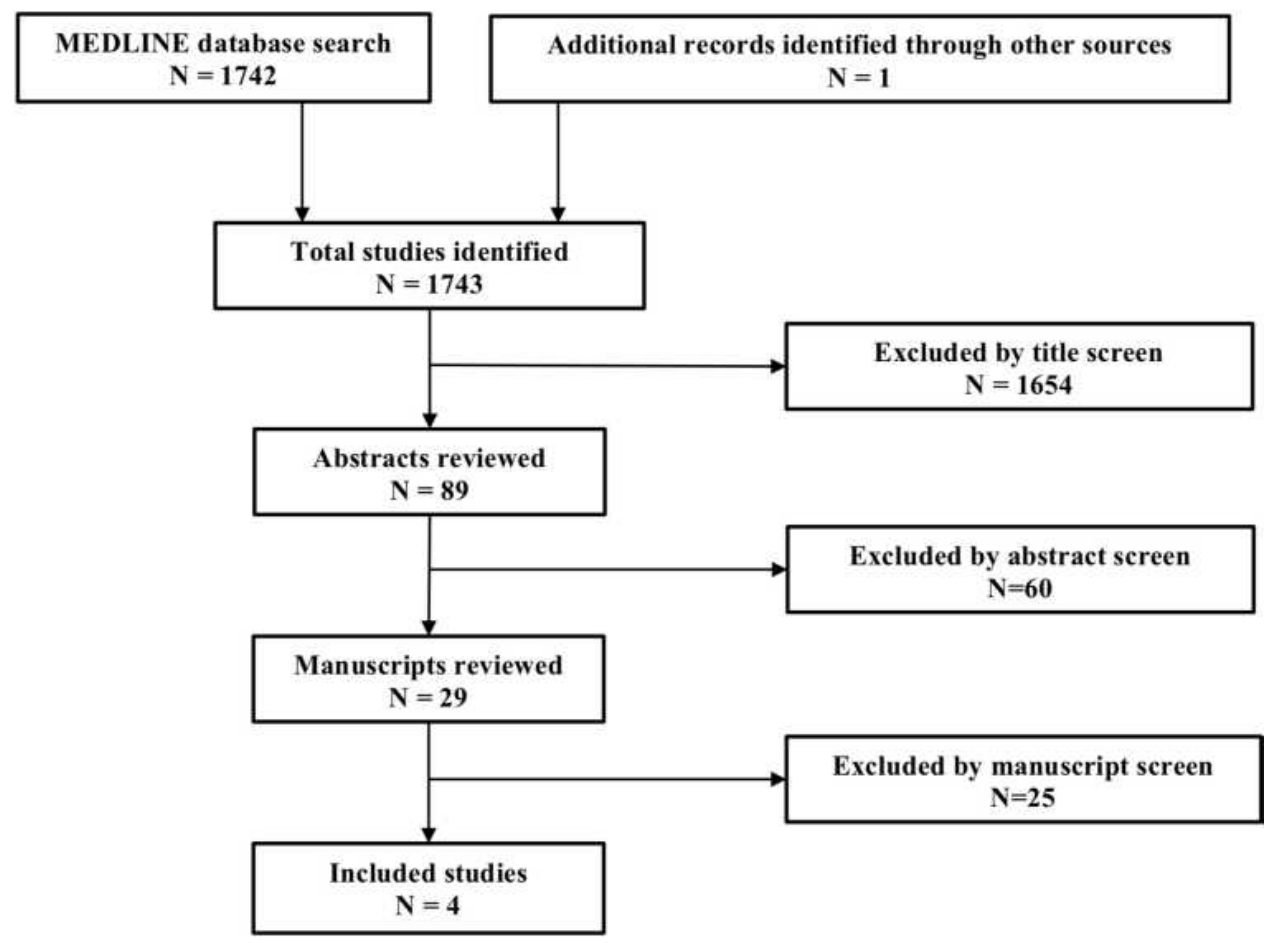

Figure I Flowchart of included studies.

\section{Discussion}

In athletes, the goal of physical rehabilitation following operative and nonoperative management of $\mathrm{AC}$ joint injuries is to return to the previous level of play with minimal symptomology in a safe and timely manner. Nonoperative versus operative management of AC joint injuries, and the biomechanical analysis of numerous reconstructive techniques, has been thoroughly investigated. ${ }^{8-10,21-26}$ However, little knowledge exists pertaining to the most effective rehabilitation methodology. This insight is further encumbered by the heterogeneity of proposed rehabilitation protocols, often formulated by individual surgeon or therapist experience, with limited supporting evidence. In particular, there are no randomized controlled trials investigating the efficacy of different rehabilitation protocols and there are limited comparative studies investigating the effects of rehabilitation. Over the past 25 years, various rehabilitation protocols have been proposed but are limited in their lack of evidence-based medicine, biologic basis, or reliable control groups to reduce confounding factors or differentiate from the natural history of the disease process. Although standardization of rehabilitation protocols amongst patients can be considered experimentally appropriate, it is unsuitable for addressing individual patient goals, symptoms, and deficiencies; instead, therapy should be tailored to each individual patient. Protocols are exhaustive, homogenous, and operate under the assumption that patients progress along a uniform healing pathway. Adherence to protocols diminishes the need for clinical reasoning, critical thinking, and quality of patient care. This article serves as a reminder that rehabilitation protocols have less practicality and efficacy when used as rigid instructional regimens, but rather should be a patient-specific toolbox to guide individual shortcomings.

The AC joint is stabilized by static and dynamic components, for which the latter may be greatly impacted by effective rehabilitation. ${ }^{16,27,28}$ Some hypothesize that the deltoid-trapezius complex works in synchrony to provide dynamic stability to the AC joint. ${ }^{16,29}$ This equilibrium is disrupted with $\mathrm{AC}$ joint injuries. In fact, Lizaur et al identified that $94 \%$ of patients with AC joint separations had injury to the trapezius and/or deltoid. ${ }^{30}$ Gladstone et al emphasized the importance of adhering to rehabilitation protocols for these injuries to improve outcomes as the soft tissue injury is more extensive than can be appreciated on radiographs and "muscular injury can rapidly lead to weakness and functional deficits, secondary to pain, instability, and reflex inhibition of muscular activity."17 Development and maintenance of periscapular control 
Table I Rehabilitation Protocols Addressing AC Joint Injuries

\begin{tabular}{|c|c|c|c|}
\hline Study & Indication & Protocol & Rationale/Evidence \\
\hline $\begin{array}{l}\text { Gladstone } \\
\text { et al }(1997)^{17}\end{array}$ & $\begin{array}{l}\text { Nonoperative } \\
\text { protocol specific to } \\
\text { Types I-III AC joint } \\
\text { injuries. } \\
\text { Applicable to } \\
\text { postoperative care } \\
\text { as well. }\end{array}$ & $\begin{array}{l}\text { Type I } \\
\text { Sling ( } 5-7 \text { days) } \\
\text { Ice ( } 48 \text { hours) } \\
\text { NSAIDs } \\
\text { Gentle ROM and isometric exercises immediately } \\
\text { Strengthening as pain improves (deltoid, trapezius, } \\
\text { periscapular muscles, rotator cuff) } \\
\text { Return to sport (I-2 weeks) } \\
\text { Type II } \\
\text { Sling (I-2 weeks) } \\
\text { Protocol same as Type I } \\
\text { Return to sport (2-3 weeks) } \\
\text { Type III } \\
\text { Sling (I-4 weeks), ice, NSAIDs } \\
\text { Gentle ROM and isometric exercises (within 3-7 days) } \\
\text { No straps or tape } \\
\text { Resistance exercise program (within 2-3 weeks) - dynamic shoulder girdle stabilization } \\
\text { Return to sport (4-6 weeks) } \\
\text { Rehabilitation is divided into } 4 \text { Phases which are similar for Types I, II, and III, but progression is } \\
\text { slower in Type III injuries }\end{array}$ & $\begin{array}{l}\text { ROM nourishes articular } \\
\text { cartilage, supports collagen } \\
\text { synthesis/organization, and allows } \\
\text { neuromodulation of pain. } \\
\text { Isokinetic strength profiles used } \\
\text { as a guide to determine return to } \\
\text { competition readiness. }\end{array}$ \\
\hline $\begin{array}{l}\text { Cote et al } \\
(2010)^{18}\end{array}$ & $\begin{array}{l}\text { Operative and } \\
\text { nonoperative } \\
\text { protocols for Type } \\
\text { I-VI AC joint } \\
\text { injuries }\end{array}$ & $\begin{array}{l}\text { Nonoperative - modeled after the Gladstone protocol } \\
\text { Type I } \\
\text { Early ROM (Ist week) } \\
\text { Avoid internal rotation, cross-body adduction, end-ROM for Ist week } \\
\text { Strengthening - progress as tolerated } \\
\text { CKC exercises } \\
\text { Isotonic \& OKC exercises } \\
\text { Function-specific exercises } \\
\text { Return to sport ( } 2 \text { weeks) } \\
\text { Type II } \\
\text { Immobilization } \\
\text { Scapular retraction } \\
\text { CKC exercises } \\
\text { OKC exercises } \\
\text { Return to full activity once able to perform task-specific activities } \\
\text { Type III } \\
6 \text {-I2-week rehabilitation trial } \\
\text { Same protocol as Type II } \\
\text { Early lower extremity and core exercises } \\
\text { Scapular retraction brace } \\
\text { Types IV-VI } \\
\text { Same protocol as Type III } \\
\text { Operative - after ACCR } \\
\text { Platform brace (6-8 weeks) } \\
\text { Active-assisted ROM } \\
\text { CKC exercises (at } 8 \text { weeks) } \\
\text { Isotonic \& OKC exercises (I2-I8 weeks) }\end{array}$ & $\begin{array}{l}\text { Electromyography support of } \\
\text { exercises }\end{array}$ \\
\hline
\end{tabular}

Abbreviations: AC, acromioclavicular; ACCR, anatomic coracoclavicular ligament reconstruction; ACLC, acromioclavicular ligament complex; CKC, closed kinetic chain; NSAIDs, non-steroidal anti-inflammatory drugs; OKC, open kinetic chain; ROM, range of motion.

and stabilization of the shoulder girdle is the foundation for nonoperative management of AC joint ligamentous injuries, allowing more rapid return to activity, ${ }^{17}$ irrespective of injury grade. Even in those with chronic Type III AC joint injuries, Carbone et al identified $78 \%$ resolution of scapular dyskinesis 
Table 2 Rehabilitation Protocols Addressing Scapular Dyskinesis

\begin{tabular}{|c|c|c|c|}
\hline Study & Indication & Protocol & Rationale/Evidence \\
\hline $\begin{array}{l}\text { Kibler and } \\
\text { McMullen } \\
(2003)^{19}\end{array}$ & $\begin{array}{l}\text { Scapular dyskinesis; } \\
\text { not specific to AC } \\
\text { joint injuries }\end{array}$ & $\begin{array}{l}\text { Acute Phase ( } 0-3 \text { weeks) } \\
\text { Decrease pain } \\
\text { Proximal facilitation (hip and trunk motions) } \\
\text { Soft tissue mobilization } \\
\text { Assisted stretching } \\
\text { CKC exercises } \\
\text { Scapular motions without arm elevation } \\
\text { Incorporate arm motion } \\
\text { Recovery Phase ( } 2-8 \text { weeks) } \\
\text { CKC exercises with increased load and elevation } \\
\text { Arm elevation and rotation } \\
\text { OKC exercises } \\
\text { Lunges with dumbbell reaches } \\
\text { Maintenance Phase (6-I0 weeks) } \\
\text { Plyometric exercises } \\
\text { Dumbbell presses and punches }\end{array}$ & $\begin{array}{l}\text { Altered periscapular force coupling secondary to } \\
\text { changes in muscle inhibition and activation patterns } \\
\text { following injury } \\
\text { Kinetic chain alterations } \\
\text { Biomechanical alterations in glenohumeral contact } \\
\text { position }\end{array}$ \\
\hline $\begin{array}{l}\text { Burkhart } \\
\text { et al } \\
(2003)^{20}\end{array}$ & $\begin{array}{l}\text { Scapular dyskinesis; } \\
\text { not specific to AC } \\
\text { joint injuries }\end{array}$ & $\begin{array}{l}\text { Goals: Stretching \& Strengthening } \\
\text { Stretching: } \\
\text { Pectoralis minor } \\
\text { Rolled towel along spine of supine patient, push } \\
\text { shoulders posteriorly } \\
\text { Posteroinferior capsule } \\
\text { "Sleeper stretch" } \\
\text { Strengthening: } \\
\text { Scapular protraction, retraction, depression, } \\
\text { elevation, rotation } \\
\text { CKC exercises } \\
\text { OKC lunges + diagonal pulls } \\
\text { Blackburn exercises } \\
\text { Seated push-ups } \\
\text { Rowing, both standard and low row }\end{array}$ & $\begin{array}{l}\text { Muscular imbalances } \\
\text { Kinetic chain alterations } \\
\text { Biomechanical alterations to the glenohumeral and } \\
\text { scapulothoracic joints }\end{array}$ \\
\hline
\end{tabular}

Abbreviations: AC, acromioclavicular; CKC, closed kinetic chain; OKC, open kinetic chain.

following detailed scapular rehabilitation, which may have been avoidable if periscapular rehabilitation was stressed in the acute setting. ${ }^{14,20}$

Current rehabilitation protocols specifically for AC joint injuries describe four phases of rehabilitation, with variations in timing, activity restrictions, and exercises based on injury grade. ${ }^{17,18}$ There is a gradual progression from immobilization to range of motion, strengthening, and sports-specific training. Although not specific to AC joint injuries, protocols designed for treating SICK scapula syndrome and scapular dyskinesis, such as those presented by Kibler and McMullen et al and Burkhart et al, are useful in assisting in scapular stabilization following AC joint injuries. ${ }^{19,20}$

\section{Our Rehabilitation Philosophy}

In our experience, the foundation of $\mathrm{AC}$ rehabilitation is periscapular control. This can be accomplished with a stepwise approach involving pain reduction for tolerance of activities, mobilization to permit adequate muscle recruitment, periscapular muscle strengthening, and finally, sport-specific exercises. Current protocols are exhaustive, portraying inflexible rehabilitation regimens that limit critical reasoning and thinking by the therapist. Furthermore, many rehabilitation protocols are adjusted based on injury grade, eg, Rockwood classification; however, we have found that therapists have limited utility in applying these radiographic findings to guide 
individualized treatment. Consequently, previously published protocols should be simplified to allow individualized treatment options for the patient. Moreover, effective communication between physical therapists and physicians is imperative. ${ }^{31}$ Established multidisciplinary rapport allows individualized barriers to be addressed and conveyed to all members of the treatment team so that therapist decisions are seen as intuitive and constructive rather than a failure to adhere to the physician-prescribed treatment plan. Our current rehabilitation framework involves 4 phases. Progression between these phases is patientdependent and should be geared toward individualized symptoms and goals, rather than arbitrary time points and motion thresholds.

Phase 1 involves a brief period of immobilization for pain alleviation, to allow early rehabilitation and cease disproportionate guarding. Patients are typically first seen in the emergency or urgent care setting and are provided with a sling to be worn until their first outpatient appointment. Sling immobilization supports the limb, decreasing stresses on the AC joint, and assists with inflammation reduction, allowing pain to subside. For the majority of patients, immobilization lasts for approximately 3-10 days and the patient can selfdiscontinue when they find it appropriate. This often occurs when patients are free of pain with the arm at the side and during activities of self-care. Unfortunately, prolonged immobilization can lead to a slower recovery and risk of disuse atrophy. ${ }^{17,32}$ Rehabilitation has been shown to rapidly suppress and sustain below basal levels of myostatin, a protein that inhibits muscle differentiation and growth in vivo, thus promoting injury recovery. ${ }^{32}$ Moreover, early range of motion can help decrease inflammation and pain. ${ }^{18}$

Phase 2 involves range of motion exercises and early strengthening. In the early stages of rehabilitation, pain free range of motion is obligatory for later functional strengthening. Patients begin with passive and activeassisted range of motion exercises, particularly working on external rotation and forward elevation to shoulder level, which may be limited following prolonged sling immobilization. Activities such as internal rotation behind the back, cross-body adduction, and extremes of forward elevation are often avoided early on, as they tend to provoke symptoms. However, these motions can be initiated if well tolerated. Patients may also begin active-assisted range of motion (AAROM). In our experience, active ROM of the affected shoulder may elicit pain and could cause regression back to a sling. Instead, we recommend closed-chain scapular exercises to allow early scapular motion and muscle recruitment. Closed-chain exercises, such as scapular clocks (Figure 2) and isometric periscapular exercises, reduce $\mathrm{AC}$ joint stress by unloading the weight of the arm, allowing the patient to focus on scapular motion, particularly scapular retraction. ${ }^{33}$

Phase 3 incorporates advanced dynamic strengthening of the shoulder girdle and periscapular musculature for improvement of AC joint stability. Closed-chain exercises are continued and patients can progress to tubing or cable exercises to further strengthen the periscapular muscles and rotator cuff. Scapular retraction can be improved with rowing or tubing exercises. To follow, kinetic chain (eg, disco motions) and open-chain exercises are begun to further promote scapular stability. Electromyography studies have identified Blackburn exercises (eg, "Ts" and "Ys") as desirable, recruiting high levels of muscle activity of the middle and lower trapezius, however, they can place a substantial stress on the AC joint secondary to a long lever arm. ${ }^{18,34,35}$ As such, these exercises should be avoided early in the rehabilitation process. Typical Phase 3 exercises are shown in Figure 3.

Phase 4 integrates sports-specific training. Kinetic chain exercise, plyometric activity, and strengthening are advanced. Kinetic chain exercises to recruit core, trunk, and leg musculature will facilitate force coupling and periscapular activation to the scapulothoracic and glenohumeral joints. ${ }^{19}$ For power athletes such as defensive linemen, pushups, bench press and overhead press can be begun, preventing lockout to limit stress to the healing AC joint. Using a medicine ball, two-hand chest passes can be started with progression to single-hand activities. For throwers, a graduated throwing program is initiated, starting at short distances and low velocity and advancing to increased number of throws, longer distances, and balls thrown more on a direct line at higher velocity. For other overhead athletes such as racquet sports players, ground strokes and then overhead service can be initiated depending on the tolerance of symptoms.

However, the progression of these phases is not uniform and should be seen as a continuum, rather than arbitrary thresholds for advancement. At our institution, we have identified 3 common barriers in our patient population that hinder effective rehabilitation: Pain, Apprehension, and (anterior chest wall) Stiffness; and 

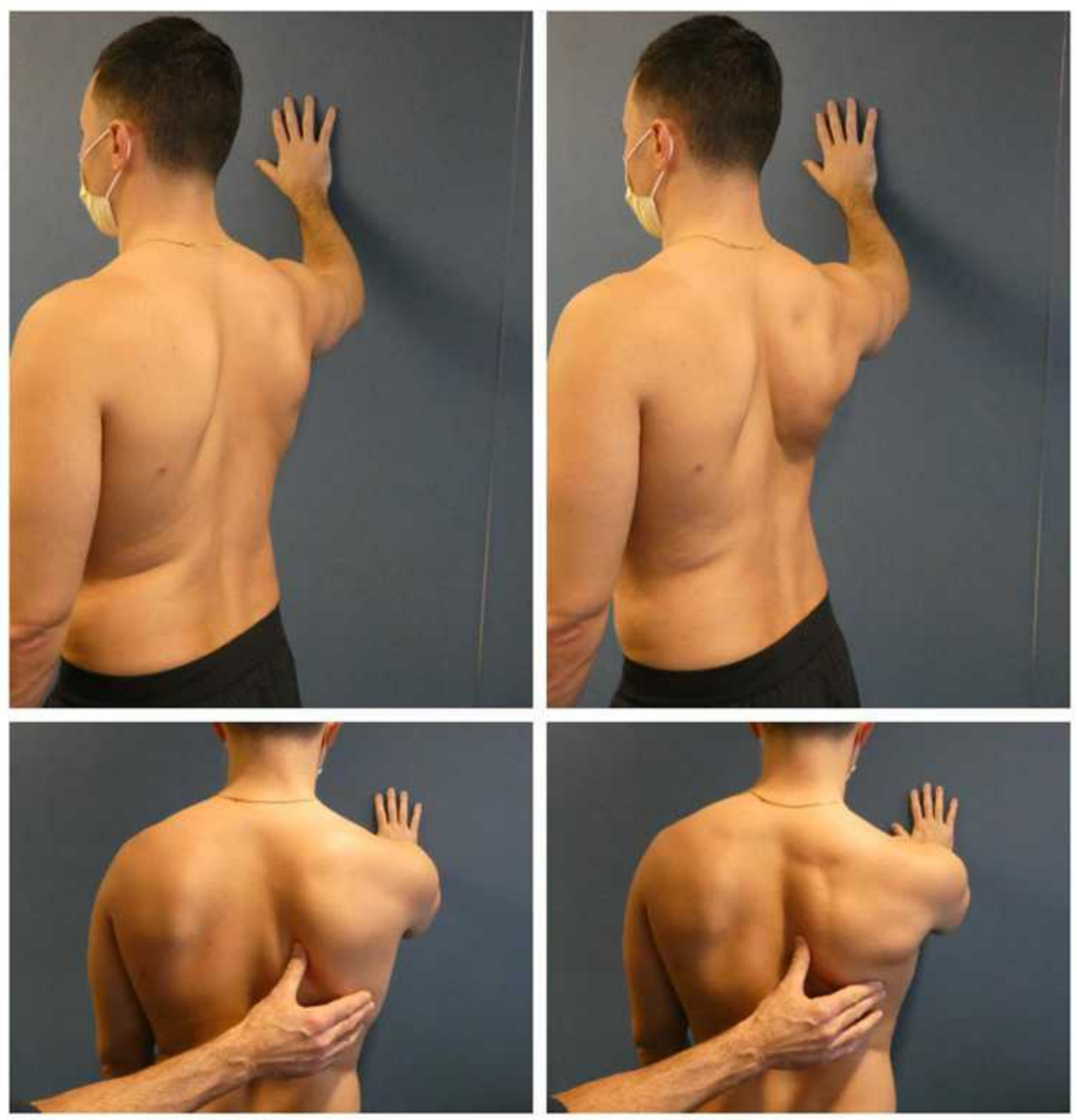

Figure 2 Scapular clocks are effective closed-chain exercises that allow recruitment of the periscapular musculature, while limiting AC joint stress by unweighting the arm. Top, directing the affected scapula to the contralateral pant pocket with sustained contraction helps emphasize scapular retraction. Bottom, the examiner's hand placed at the inferomedial border of the scapula is helpful for tactile feedback.

consequently, precluding Scapular control ("PASS"). Identification of these individualized patient perceptions and restrictions have warranted non-universal rehabilitation principles in our patient population.

\section{Pain}

Severe, persistent pain that outlasts both brief immobilization and other conservative measures, including cryotherapy and anti-inflammatory medications, presents difficulties for effective rehabilitation. These patients are often reluctant to initiate therapy and opt for continued immobilization. However, it is critical to avoid prolonged immobilization as it can result in disuse atrophy and stiffness. Early rehabilitation should focus on pain alleviation and gentle ROM. Overaggressive rehabilitation by the therapist can lead to regression with prolonged sling use and increased guarding. Severe muscle guarding must be addressed early with gentle, passive, therapistdirected forward flexion and external rotation. If muscle guarding persists, active-assisted motion by the patient using their contralateral arm for support and control can improve comfort, increase confidence, and provide reassurance to the patient while initiating therapy. Provocative maneuvers including cross-body adduction, internal rotation behind the body, and end-ROM should be avoided until the pain has decreased. Furthermore, active ROM should be delayed, as the long lever arm and the weight of the upper extremity can stress the AC joint, worsening the pain.

After muscle guarding has ceased, closed-chain scapular clocks and isometric exercises are helpful to further ROM and periscapular muscle recruitment, while advantageously unloading the arm. Scapular clock exercises can begin with the hand at table level with gradual 

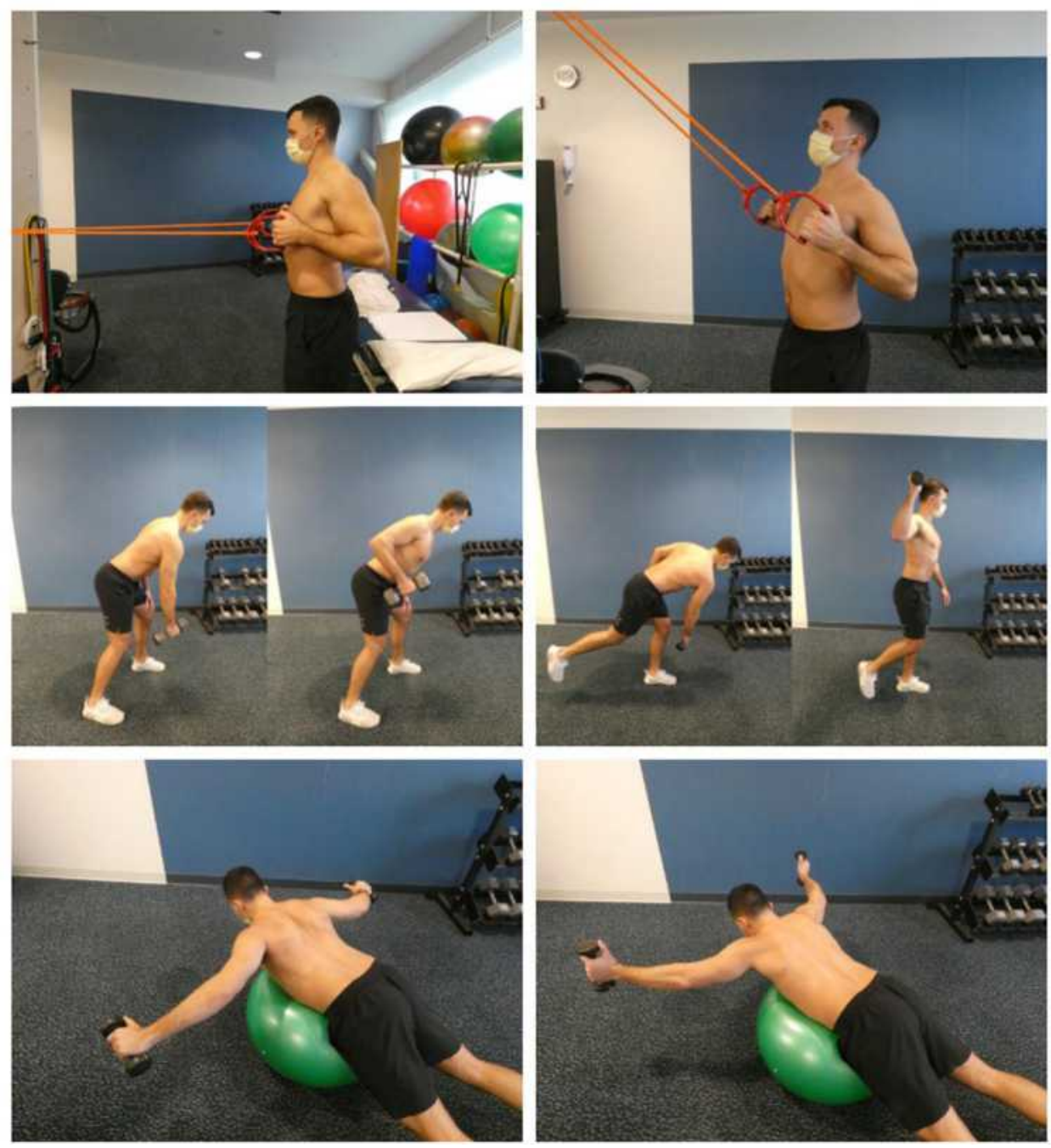

Figure 3 Phase 3 involves periscapular and rotator cuff strengthening. Top, rowing exercises with resistance tubing at various level of forward elevation are helpful, but the patient needs to focus on scapular retraction rather than compensation with shoulder extension. Middle, kinetic chain exercises including lawn mower and disco exercises help re-build functional strength. Bottom, Blackburn exercises, also called "T's and "Y's" are advanced strengthening exercises, recruiting high levels of middle and lower trapezius, at the expense of high stress placed at the AC joint. These are generally reserved for later in the rehabilitation process.

increases in forward elevation dependent on patient symptoms. Patients should focus on directing the scapular to the contralateral pants pocket to promote muscle recruitment and control, rather than strengthening. Similarly, isometric low row exercises focusing on scapular retraction are helpful. The examiner or the patient, using their contralateral arm, can place a hand at the midline of the thoracic spine to use as a goal for positive reinforcement. Helpful exercises for patients in acute pain are shown in Figure 4.

\section{Apprehensive}

Apprehensive patients are different from those with acute pain. These patients have had improvements in pain, but advancements are hindered by subjective symptoms and objective alterations in upper extremity kinematics and strength. Patients may experience clicking, fatigue, and weakness, which may elicit feelings that these activities are inappropriate and even destructive. Patients may also be concerned with visual asymmetries of their shoulder girdle or an apparent "upper extremity limp;" the latter a result of asynchrony between the trunk, scapula, and arm secondary to muscular imbalance and/or pain. These patients often require more counseling and stepwise, goal-directed therapy. Similar to the acutely painful patient, we have found success with passive ROM and closed chain exercises that unweight the arm. By removing gravitational forces from the arm, therapy can be continued while patients regain the confidence to mobilize the shoulder. These patients benefit 

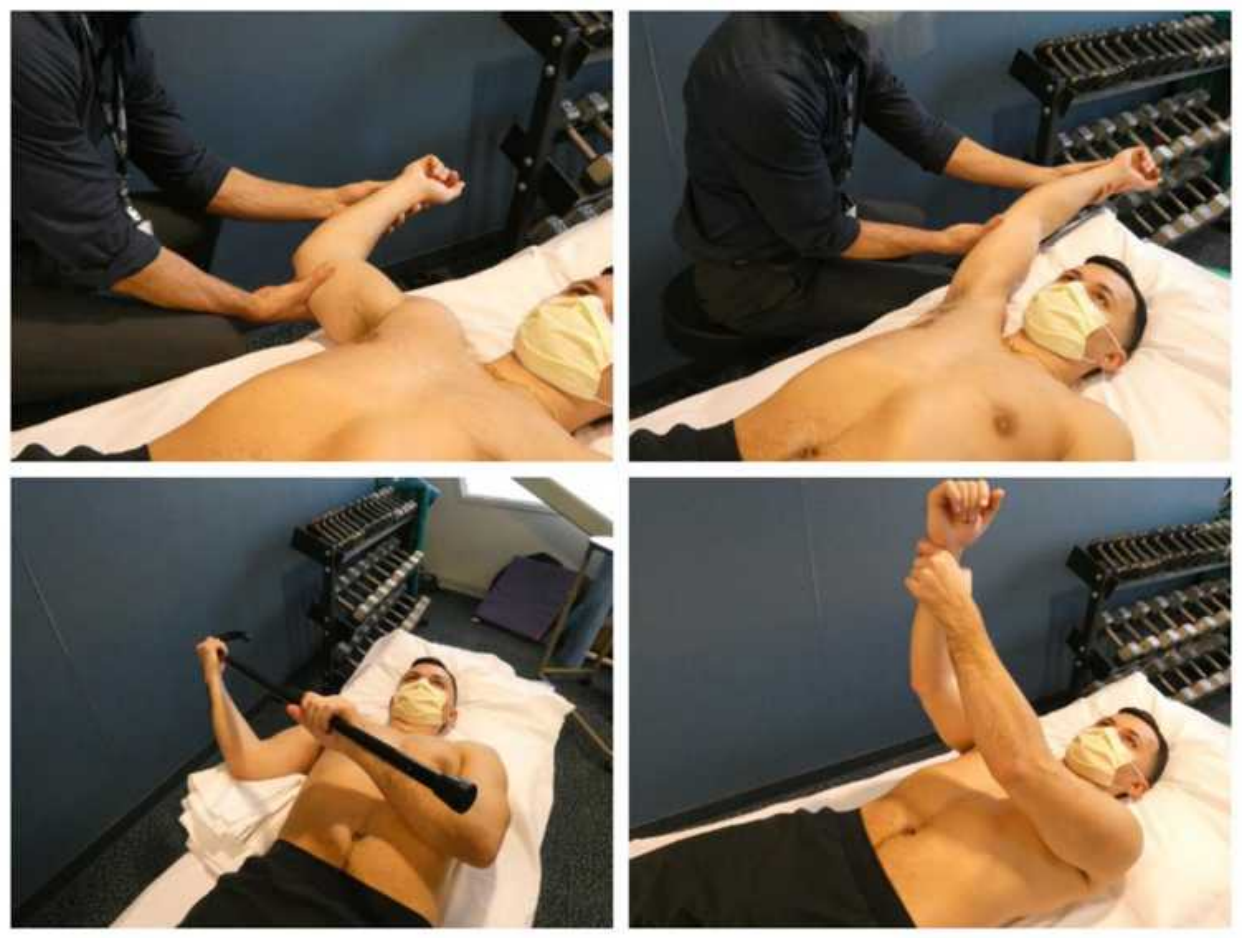

Figure 4 Patients in acute pain benefit from gentle range of motion (top) and active-assisted range of motion (bottom) working on external rotation and forward elevation. Active-assisted motion can be moderated using a cane/L-bar or the patient can use their contralateral upper extremity.

from slow, deliberate motions to restore confidence and improve unwieldy post-injury kinematics. Table slide exercises focusing on sustained scapular retraction are advisable. These exercises are started on flat surfaces and as patients advance, inclined surfaces or activities further from the body can be initiated (Figure 5).

\section{Anterior Chest Wall Stiffness}

Patients immobilized for a prolonged period may develop significant pectoralis minor stiffness or anterior chest wall stiffness. These patients present with a hunched shoulder that is adducted and internally rotated, with a protracted scapula and thoracic spine kyphosis. ${ }^{20}$ Depending on the length of immobilization, muscular atrophy may be present.

Rehabilitation begins with immediate sling removal. Patients are positioned supine with a rolled towel placed along the thoracic spine. In patients with anterior chest wall stiffness, the examiner will be able to identify asymmetry of the shoulders, with the affected shoulder further elevated off the examining table. The towel assists with scapular retraction as gravitational forces help to retract the shoulders. In this position, soft tissue manipulation and massage can be applied to the pectoralis minor insertion at the coracoid. Additionally, therapist-driven stretching and manipulation can help to release the anterior contraction and allow progression of therapy. While standing at the side of the table, the therapist can provide sustained manual pressure downwards on the affected shoulder to help retraction (Figure 6).

Following improvements in anterior stiffness, scapular retraction exercises (eg, low cable rows, scapular clocks) are begun. Patients should focus on maintaining an open anterior thorax in addition to scapular retraction. During isometric low cable rows, it is important to encourage the patient to retract the scapula, rather than extending at the shoulder. During scapular clock exercises, the patient is instructed to move their scapula towards the contralateral pants pocket. Be mindful that patients initially tend to depress the scapula, instead of retracting. Once scapular control has been established, the therapist can add manual resistance by placing a hand at the inferomedial border of the scapula and having the patient retract against resistance.

In a like manner, mobility of the thoracic spine needs to be emphasized to improve scapulothoracic joint kinematics. Thoracic spine kyphosis following immobilization of AC joint injury leads to anterior tilting of the scapula in 

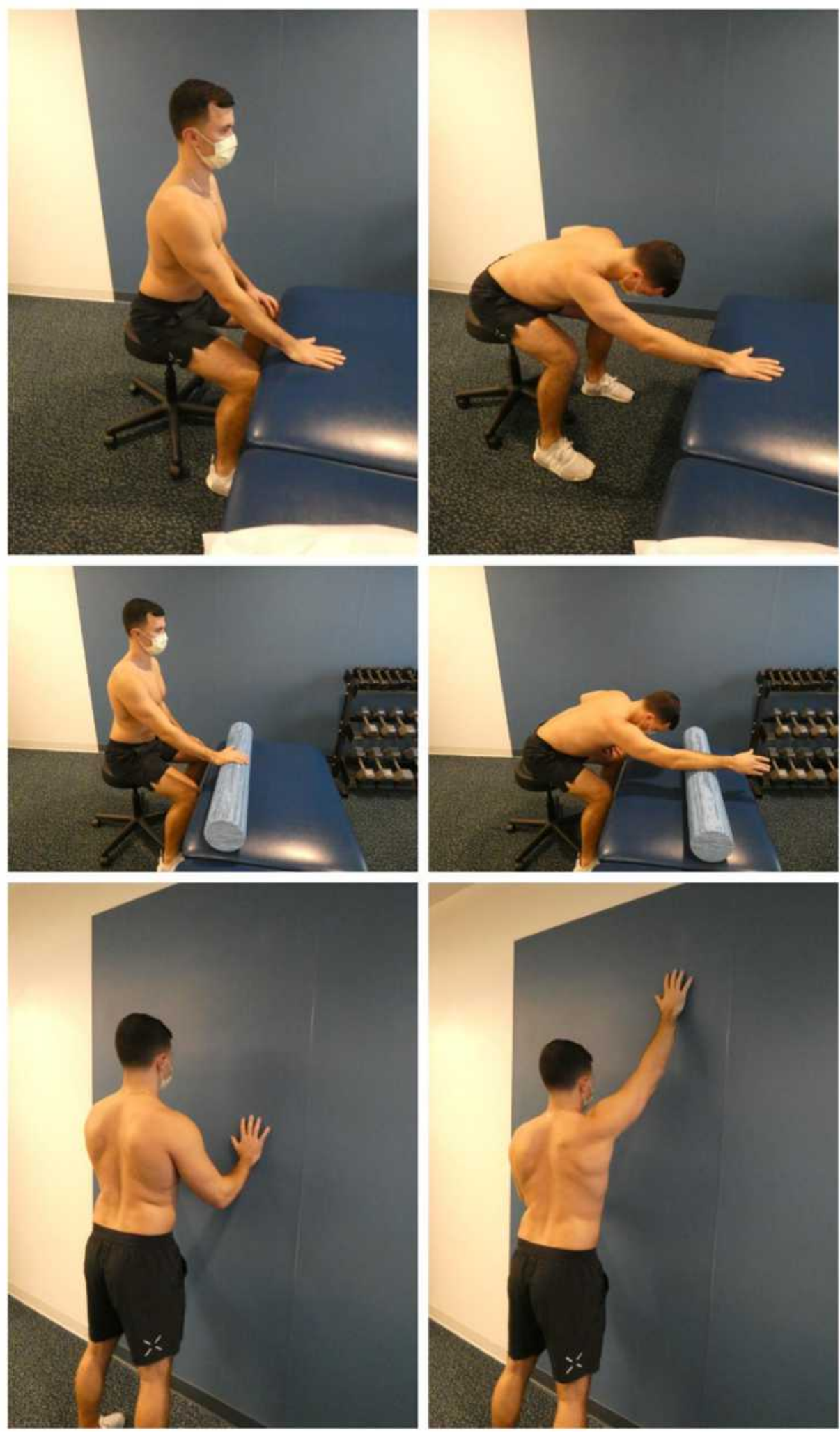

Figure 5 In those with apprehension, closed chain exercises including table slides (top), roller exercises (middle), and wall slides (bottom) unweight the arm, allowing the patient to isolate and focus on periscapular muscle activation. With the arm in a fixed position, asynchronies between the trunk, scapula, and arm are minimized. 

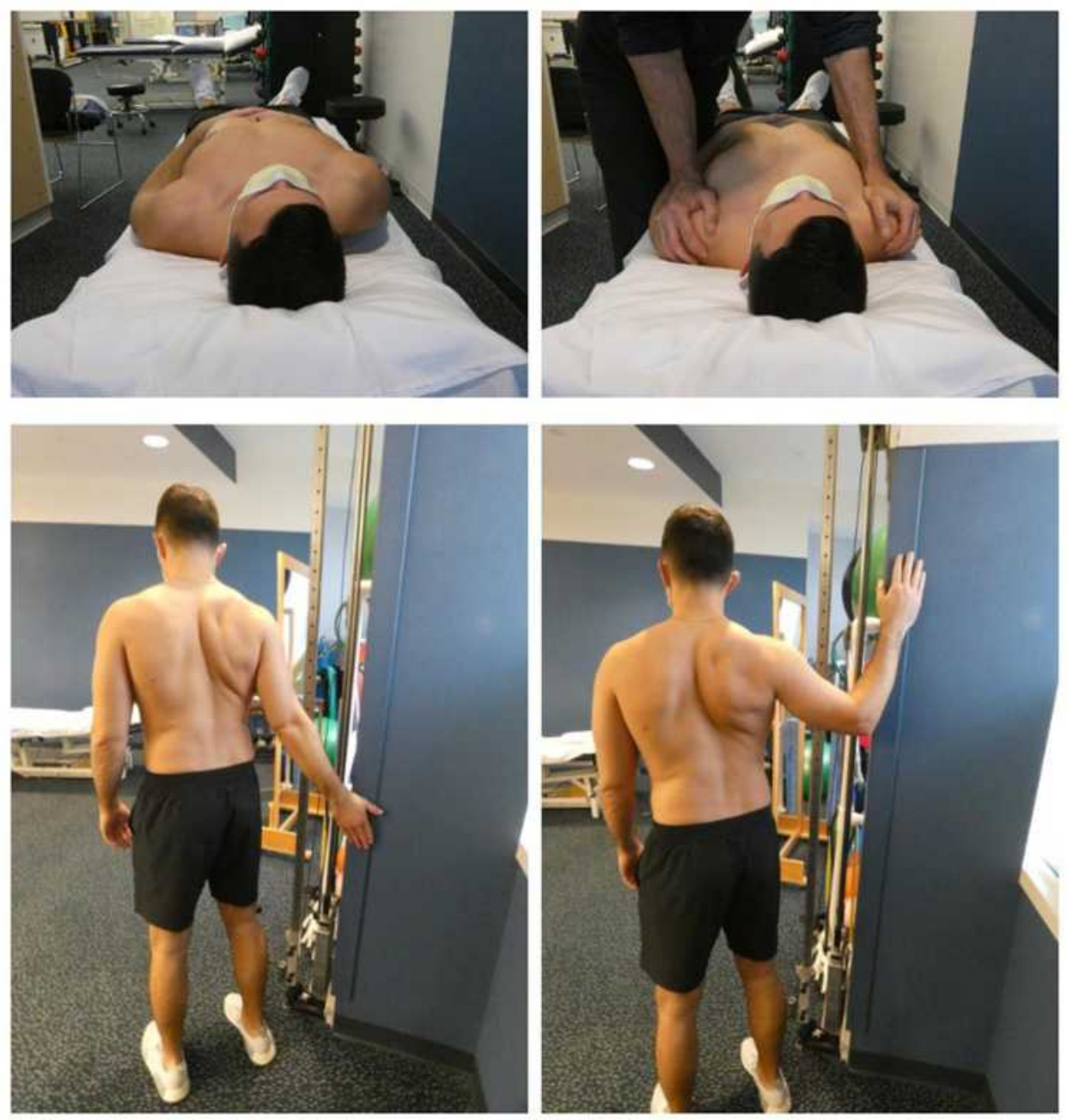

Figure 6 Anterior chest wall stiffness results in a protracted scapula with limitations on functional range of motion. Top, a rolled up towel can be placed at the midline of the thoracic spine to allow gravity-dependent scapular retraction. Asymmetry evidenced by unilateral shoulder elevation off the examination table can be mitigated using slow and sustained manual downward pressure by the therapist. Bottom, various stretching exercises for pectoralis minor stiffness should be utilized while focusing on scapular retraction.

the sagittal plane, limiting improvements in forward elevation, abduction, and horizontal adduction during rehabilitation.

Overall, strict rehabilitation protocols with rigid, arbitrary timelines limit critical thinking and reasoning by therapists, failing to optimize rehabilitation for athletes following acromioclavicular joint injuries. As such, effective communication and favorable, trusting relationships between physicians and therapists are mandatory. Rehabilitation efforts need to be patient-specific to address individual shortcomings. In our experience, the most consistent obstacles in regaining scapular control that we identify are pain, apprehension, and anterior chest wall stiffness. The authors have provided specific tools to address these patient presentations, in order to advance athletes in their rehabilitation efforts.

\section{Conclusion}

The foundation of rehabilitation following acromioclavicular injuries is restoration of scapular control to permit safe return to sport and high-level activities. Coordinated and efficient scapular motion can be re-established through a progression of pain reduction, mobilization to permit adequate muscle recruitment, periscapular muscle strengthening, and finally, sport-specific exercises. Oftentimes, clinicians encounter barriers to this progression, for which standardized, rigid rehabilitation protocols fail to allow constructive treatment adaptation that would get an individual patient on a new path to recovery. Three common barriers preventing successful acromioclavicular injury rehabilitation were identified and addressed: Pain, Apprehension, and (anterior chest wall) Stiffness; preventing appropriate Scapular control, effectively termed 
"PASS". Pain can be addressed through gentle and activeassisted range of motion and closed chain exercises to limit AC joint stress and to prevent voluntary guarding that would otherwise delay periscapular muscle activation (Figure 4). Apprehension can be negotiated by first establishing proximal control using closed chain exercises as well as application of slow and deliberate motions to limit subjective discomforts of altered upper extremity motion (Figure 5). Anterior chest wall stiffness can be managed through pectoralis minor stretching and thoracic spine mobility to place the scapula in an appropriate position for upper extremity motion (Figure 6). By addressing these barriers and the bio-psycho-social aspects of injury, the quality of patient care can be improved.

\section{IRB Approval}

Institutional review board (IRB) approval was not indicated for this systematic review.

\section{Copyright/Ethics}

The actor in the figures has provided informed consent for the images to be published.

\section{Author Contributions}

All authors contributed to data analysis, drafting or revising the article, have agreed on the journal to which the article will be submitted, gave final approval of the version to be published, and agree to be accountable for all aspects of the work.

\section{Disclosure}

LeVasseur MR, Mancini MR, Berthold DP, Cusano A, McCann GP, and Gomlinski G declare that they have no conflict of interest.

Cote MP receives personal fees from Arthroscopy Association of North America (AANA) outside of the submitted work. Mazzocca AD reports research grants from Arthrex Inc. outside the submitted work and is a consultant for Arthrex Inc. The authors report no other potential conflicts of interest for this work.

This investigation was performed at the Department of Orthopaedic Surgery, University of Connecticut, Farmington, Connecticut, USA.

\section{References}

1. Bishop JY, Kaeding C. Treatment of the acute traumatic acromioclavicular separation. Sports Med Arthrosc. 2006;14:237-245. doi:10.1097/01.jsa.0000212330.32969.6e
2. Kaplan LD, Flanigan DC, Norwig J, Jost P, Bradley J. Prevalence and variance of shoulder injuries in elite collegiate football players. $\mathrm{Am}$ J Sports Med. 2005;33:1142-1146. doi:10.1177/0363546505274718

3. Kelly BT, Barnes RP, Powell JW, Warren RF. Shoulder injuries to quarterbacks in the National Football League. Am J Sports Med. 2004;32:328-331. doi:10.1177/0363546503261737

4. Chillemi C, Franceschini V, Dei Giudici L, et al. Epidemiology of isolated acromioclavicular joint dislocation. Emerg Med Int. 2013;2013:1-5. doi:10.1155/2013/171609

5. Rockwood CA, Williams GR, Young DC. Disorders of the acromioclavicular joint. In: Rockwood CA, Matsen FA, editors. The Shoulder. Philadelphia: Saunders; 1998:483-553.

6. Warth RJ, Martetschläger F, Gaskill TR, Millett PJ. Acromioclavicular joint separations. Curr Rev Musculoskelet Med. 2013;6(1):71-78. doi:10.1007/s12178-012-9144-9

7. Johansen JA, Grutter PW, McFarland EG, et al. Acromioclavicular joint injuries: indications for treatment and treatment options. J Shoulder Elb Surg. 2011;20:70-82. doi:10.1016/j.jse.2010.10.030

8. Beitzel K, Cote MP, Apostolakos J, et al. Current concepts in the treatment of acromioclavicular joint dislocations. Arthroscopy. 2013;29:387-397. doi:10.1016/j.arthro.2012.11.023

9. McFarland EG, Blivin SJ, Doehring CB, et al. Treatment of grade III acromioclavicular separations in professional throwing athletes: results of a survey. Am J Orthop. 1997;26:771-774.

10. Nissen CW, Chatterjee A. Type III acromioclavicular separation: results of a recent survey on its management. Am J Orthop. 2007;36:89-93.

11. Neer CS. Shoulder Reconstruction. Philadelphia, PA: Saunders; 1990:341-355.

12. Pavlatos CJ. Acromioclavicular joint injuries. In: Andrews JR, Wilk KE, editors. The Athlete's Shoulder. New York, NY: Churchill Living- stone; 1994:291-303.

13. Murena L, Canton G, Vulcano E, Cherubino P. Scapular dyskinesis and SICK scapula syndrome following surgical treatment of type III acute acromioclavicular dislocations. Knee Surg Sports Traumatol Arthrosc. 2013;21:1146-1150. doi:10.1007/s00167-012-1959-9

14. Carbone S, Postacchini R, Gumina S. Scapular dyskinesis and SICK syndrome in patients with a chronic type III acromioclavicular dislocation. Results of rehabilitation. Knee Surg Sports Traumatol Arthrosc. 2015;23:1473-1480. doi:10.1007/s00167-014-2844-5

15. Gumina S, Carbone S, Postacchini F. Scapular dyskinesis and SICK scapula syndrome in patients with chronic type III acromioclavicular dislocation. Arthrosc - J Arthrosc Relat Surg. 2009;25:40-45. doi:10.1016/j.arthro.2008.08.019

16. Reid D, Polson K, Johnson L. Acromioclavicular joint separations grades IIII: a review of the literature and development of best practice guidelines. Sport Med. 2012;42:681-696. doi:10.1007/ BF03262288

17. Gladstone JN, Wilk KE, Andrews JR. Nonoperative treatment of acromioclavicular joint injuries. Oper Tech Sports Med. 1997;5:78-87. doi:10.1016/S1060-1872(97)80018-4

18. Cote MP, Wojcik KE, Gomlinski G, Mazzocca AD. Rehabilitation of acromioclavicular joint separations: operative and nonoperative considerations. Clin Sports Med. 2010;29:213-228. doi:10.1016/j. csm.2009.12.002

19. Kibler BW, McMullen J. Scapular dyskinesis and its relation to shoulder pain. J Am Acad Orthop Surg. 2003;11:142-151. doi:10.5435/00124635-200303000-00008

20. Burkhart SS, Morgan CD, Kibler WB. The disabled throwing shoulder: spectrum of pathology part III: the SICK scapula, scapular dyskinesis, the kinetic chain, and rehabilitation. Arthrosc - J Arthrosc Relat Surg. 2003;19:641-661. doi:10.1016/S0749-8063(03)00389-X

21. Beitzel K, Obopilwe E, Apostolakos J, et al. Rotational and translational stability of different methods for direct acromioclavicular ligament repair in anatomic acromioclavicular joint reconstruction. Am. J. Sports Med. 2014;42:2141-2148. doi:10.1177/ 0363546514538947 
22. Costic RS, Labriola JE, Rodosky MW, Debski RE. Biomechanical rationale for development of anatomical reconstructions of coracoclavicular ligaments after complete acromioclavicular joint dislocations. Am J Sports Med. 2004;32:1929-1936. doi:10.1177/ 0363546504264637

23. Deshmukh AV, Wilson DR, Zilberfarb JL, Perlmutter GS. Stability of acromioclavicular joint reconstruction: biomechanical testing of various surgical techniques in a cadaveric model. Am J Sports Med. 2004;32:1492-1498. doi:10.1177/0363546504263699

24. Grutter PW, Petersen SA. Anatomical acromioclavicular ligament reconstruction: a biomechanical comparison of reconstructive techniques of the acromioclavicular joint. Am J Sports Med. 2005;33:1723-1728. doi:10.1177/0363546505275646

25. Mazzocca AD, Santangelo SA, Johnson ST, et al. A biomechanical evaluation of an anatomical coracoclavicular ligament reconstruction. Am J Sports Med. 2006;34:236-246. doi:10.1177/0363546505281795

26. Wellmann M, Kempka JP, Schanz S, et al. Coracoclavicular ligament reconstruction: biomechanical comparison of tendon graft repairs to a synthetic double bundle augmentation. Knee Surg Sports Traumatol Arthrosc. 2009;17:521-528. doi:10.1007/s00167-009-0737-9

27. Salter EG, Nasca RJ, Shelley BS. Anatomical observations on the acromioclavicular joint and supporting ligaments. Am J Sports Med. 1987;15:199-206. doi:10.1177/036354658701500301

28. Kumar VP, Satku K, Liu J, Shen Y. The anatomy of the anterior origin of the deltoid. J Bone Joint Surg Br. 1997;79-B:680-683. doi:10.1302/0301-620X.79B4.0790680
29. Pastor MF, Averbeck AK, Welke B, et al. The biomechanical influence of the deltotrapezoid fascia on horizontal and vertical acromioclavicular joint stability. Arch. Orthop. Trauma Surg. 2016;136:513-519. doi:10.1007/s00402-015-2389-1

30. Lizaur A, Marco L, Cebrian R. Acute dislocation of the acromioclavicular joint. Traumatic anatomy and the importance of deltoid and trapezius. J Bone Joint Surg Br. 1994;76-B:602-606. doi:10.1302/ 0301-620X.76B4.8027149

31. Hulme J, Bach B, Lewis J. Communication between physicians and physical therapists. Phys Ther. 1988;68:26-31. doi:10.1093/ptj/ 68.1 .26

32. Marimuthu K, Murton AJ, Greenhaff PL. Highlighted topic signals mediating skeletal muscle remodeling by activity mechanisms regulating muscle mass during disuse atrophy and rehabilitation in humans. J Appl Physiol. 2011;110:555-560. doi:10.1152/ japplphysiol.00962.2010

33. McMullen J, Uhl TL. A kinetic chain approach for shoulder rehabilitation. J Athl Train. 2000;35:329-337.

34. Ekstrom RA, Donatelli RA, Soderberg GL. Surface electromyographic analysis of exercises for the trapezius and serratus anterior muscles. J Orthrop Sports Phys Ther. 2003;33:247-258. doi:10.2519/ jospt.2003.33.5.247

35. Blackburn TA, McLeod WD, White B, et al. EMG analysis of posterior rotator cuff exercises. Athletic Train. 1990;25:40-45.

\section{Publish your work in this journal}

Open Access Journal of Sports Medicine is an international, peerreviewed, open access journal publishing original research, reports, reviews and commentaries on all areas of sports medicine. The manuscript management system is completely online and includes a very quick and fair peer-review system. Visit http://www.dovepress. com/testimonials.php to read real quotes from published authors. 